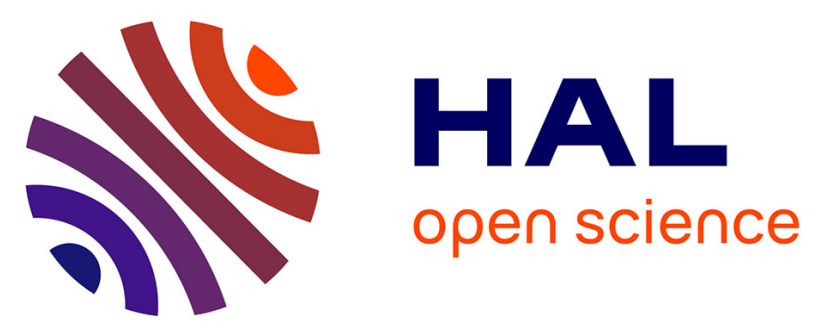

\title{
Characterisation of the C50 carotenoids produced by strains of the cheese-ripening bacterium Arthrobacter arilaitensis
}

\author{
Daniele Giuffrida, Nuthathai Sutthiwong, Paola Dugo, Paola Donato, \\ Emmanuelle Girard-Valenciennes, Francesco Cacciola, Yves Le Mao, \\ Christophe Monnet, Mireille Fouillaud, Yanis Caro, et al.
}

\section{To cite this version:}

Daniele Giuffrida, Nuthathai Sutthiwong, Paola Dugo, Paola Donato, Emmanuelle GirardValenciennes, et al.. Characterisation of the C50 carotenoids produced by strains of the cheeseripening bacterium Arthrobacter arilaitensis. International Dairy Journal, 2016, 55, pp.10-16. 10.1016/j.idairyj.2015.11.005 . hal-01399917

\section{HAL Id: hal-01399917 \\ https://hal.univ-reunion.fr/hal-01399917}

Submitted on 21 Nov 2016

HAL is a multi-disciplinary open access archive for the deposit and dissemination of scientific research documents, whether they are published or not. The documents may come from teaching and research institutions in France or abroad, or from public or private research centers.
L'archive ouverte pluridisciplinaire HAL, est destinée au dépôt et à la diffusion de documents scientifiques de niveau recherche, publiés ou non, émanant des établissements d'enseignement et de recherche français ou étrangers, des laboratoires publics ou privés. 


\title{
Characterisation of the $\mathrm{C} 50$ carotenoids produced by strains of the cheese-ripening bacterium Arthrobacter arilaitensis
}

\author{
Daniele Giuffrida a , Nuthathai Sutthiwong d, f, Paola Dugo b, c, e, Paola Donato b, c, e, \\ Francesco Cacciola ${ }^{\text {a, c }}$, Emmanuelle Girard-Valenciennes ${ }^{f}$, Yves Le Mao ${ }^{\mathrm{h}}$, \\ Christophe Monnet ${ }^{\mathrm{i}}$, Mireille Fouillaud ${ }^{\mathrm{g}}$, Yanis Caro ${ }^{\mathrm{g}}$, Laurent Dufossé ${ }^{\mathrm{g}, \mathrm{j}, \text { * }}$ \\ ${ }^{a}$ Dipartimento di Scienze dell'Ambiente, della Sicurezza, del Territorio, degli Alimenti e della Salute, Università degli Studi di Messina, Messina, Italy \\ ${ }^{\mathrm{b}}$ Dipartimento di Scienze del Farmaco e dei Prodotti per la Salute, Università degli Studi di Messina, Messina, Italy \\ c Chromaleont s.r.l., Università degli Studi di Messina, Messina, Italy \\ d Agricultural Technology Department, Thailand Institute of Scientific and Technological Research, Pathum Thani, Thailand \\ e Università Campus Bio-Medico di Roma, Centro Integrato di Ricerca, Roma, Italy \\ ${ }^{\mathrm{f}}$ Faculté des Sciences et Technologies, Université de La Réunion, Sainte-Clotilde, Reunion Island, France \\ ${ }^{g}$ ESIROI Agroalimentaire, Laboratoire de Chimie des Substances Naturelles et des Sciences des Aliments, Université de La Réunion, Sainte-Clotilde, Reunion Island, France \\ h Laboratoire de Biotechnologie agroalimentaire, Penmarc'h, Bretagne, France \\ i INRA, UMR782 Génie et Microbiologie des Procédés Alimentaires, Thiverval-Grignon, France \\ ${ }^{\mathrm{j}}$ Laboratoire ANTiOX, Université de Bretagne Occidentale, Pôle Universitaire Pierre-Jakez Hélias, F-29000 Quimper, France
}

A B S T R A C T

Pigments were extracted from cheese-ripening bacteria belonging to the species Arthrobacter arilaitensis, which plays a role in the colour of cheese. A chromatographic method was applied to characterise the pigment profile. Based on the UV-visible (UV-vis) spectra, the mass spectra, and the elution order, eight different carotenoids were identified from the strains. Four of the pigments, namely, all-E-decaprenox-anthin, all-E-sarcinaxanthin, 9-Z-decaprenoxanthin and 15$Z$-decaprenoxanthin, which were present as free-form main carotenoids (i.e., non-glycosylated), have been characterised by their UV-vis and mass spectra, both in atmospheric-pressure chemical ionisation negative [APCI (-)] and positive modes. The other minor carotenoids, namely, sarcinaxanthin monoglucoside pentaacetate, decaprenoxanthin monoglucoside, decaprenoxanthin diglucoside, decaprenoxanthin-C16:0 (decaprenoxanthin-palmitate), present in very low amounts, were only detected in the APCI $(-)$ ionisation mode. Our results provide the first chemical characterisation of the pigments produced by cheese-ripening A. arilaitensis strains, which has not previously been reported.

\section{Introduction}

Smear-ripened cheeses, also known as surface-ripened cheeses or red-smear ripened cheeses, are economically important dairy products, which involve the activity of various microorganisms during the milk coagulation and ripening stages. The surface of smear-ripened cheeses is a microbial mat, with a yellow-orangereddish-brown colour, composed of a large diversity of bacteria and yeasts. Several smear-ripened cheeses are characterised by consumers by their characteristic colour and shape, for example,

\footnotetext{
* Corresponding author. Tel.: +33262 262217544.

E-mail address: laurent.dufosse@univ-reunion.fr (L. Dufossé).
}

Livarot and Epoisses from France, Limburger and Tilsit from Germany, and Taleggio from Italy.

The colour of smear-ripened cheeses is one of the main attributes that impacts upon consumer acceptance of these cheeses, and is related to several cheese qualities, such as maturity, flavour and cleanliness (Dufossé, Mabon, \& Binet, 2001). Coloration of smear-ripened cheeses appears to be a complex phenomenon due to interactions amongst microorganisms on the cheese rind (Leclercq-Perlat, Corrieu, \& Spinnler, 2004; Mounier et al., 2006, 2008). Pigments generated by the bacteria present at the surface of cheeses are poorly studied, except for bacterial Brevibacterium species such as Brevibacterium linens and Brevibacterium aurantiacum. For a long time, these two species were considered to be the major microorganisms responsible for colour development at 
the surface of cheeses because of the ability to produce orange carotenoids, which have been identified as isorenieratene, 3hydroxy-isorenieratene, and 3,3'-dihydroxy-isorenieratene (Kohl, Achenbach, \& Reichenbach, 1983). Recently, due to the development of improved molecular methods of microbial biodiversity analysis, the presence of many other species possibly involved in the generation of functional properties has been reported in smearripened cheeses (Bockelmann \& Hoppe-Seyler, 2001; EliskasesLechner \& Ginzinger, 1995; Goerges et al., 2008; Irlinger, Layec, Hélinck, \& Dugat-Bony, 2015; Montel et al., 2014).

Arthrobacter strains have been known to be part of the cheese smear for a long time, and are now recognised as major microorganisms involved in surface pigmentation of smear-ripened cheeses due to the characteristic overall colour of their biomass and their presence during different stages of cheese production until the end of ripening (Feurer, Vallaeys, Corrieu, \& Irlinger, 2004; Irlinger \& Mounier, 2009; Larpin-Laborde et al., 2011: Mounier et al., 2005). Arthrobacter arilaitensis is frequently present at high levels on smear-ripened cheeses, and produces a yellow pigment (Galaup, Flamin, Carlet, \& Dufossé, 2005; Galaup et al., 2007; Irlinger, Bimet, Delettre, Lefèvre, \& Grimont, 2005; Leclercq-Perlat \& Spinnler, 2010; Mounier et al., 2008; Sutthiwong et al., 2014). Another species of the genus Arthrobacter, the psychrophilic bacterium Arthrobacter glacialis, was previously reported to synthesise three $\mathrm{C}_{50}$ carotenoids, i.e., decaprenoxanthin, bisanhydrobacterioruberin, and Ag470 (Arpin, Faisson, Norgard, Borch, \& Liaaen-Jensen, 1975). Arthrobacter sp. M3 was also found to produce decaprenoxanthin, and its glucosides (Arpin, Liaaen-Jensen, \& Trouilloud, 1972). In nature, C 50 carotenoids are synthesised by bacteria which belong to the Actinomycetales order. Until now, only three different $C_{50}$ carotenoid biosynthetic pathways have been described: (i) the $\beta$-cyclic $C_{50}$ carotenoid C.p. 450 pathway in Dietzia sp CQ4; (ii) the $\gamma$-cyclic $C_{50}$ carotenoid sarcinaxanthin pathway in Micrococcus luteus NCTC2665; and (iii) the $\varepsilon$-cyclic $\mathrm{C}_{50}$ carotenoid decaprenoxanthin pathway in Corynebacterium glutamicum (Heider, PetersWendisch, Netzer, Stafnes, \& Brautaset, 2014; Krubasik et al., 2001; Netzer et al., 2010; Tao, Yao, \& Cheng, 2007). The whole genome sequencing of the strain originating from cheese, A. arilaitensis Re117, has revealed the presence of a decaprenoxanthin-like carotenoid biosynthetic gene cluster (Monnet et al., 2010).

The aim of this study was to identify the pigments biosynthesised by $A$. arilaitensis strains originating from cheese to provide new information useful to elucidate the nature of the smear-ripened cheeses colour, and to understand the importance of selecting this bacterium as a part of ripening flora.

\section{Materials and methods}

\subsection{Bacterial strains, medium and growth conditions}

Two yellow-pigmented A. arilaitensis strains, namely A. arilaitensis Po102 isolated from Pont-l'Évêque cheese, and A. arilaitensis Stp101 isolated from Saint-Paulin cheese, were used in this study. These were obtained from the GMPA (Génie et Microbiologie des Procédés Alimentaires) culture collection (Unité Mixte de Recherche 782, Institut National de la Recherche Agronomique, Thiverval-Grignon, France) and maintained during this study on milk ingredient-based agar, stored at $4{ }^{\circ} \mathrm{C}$ and subcultured monthly. The milk ingredient-based agar medium contained $5 \mathrm{~g}$ casamino acids (Difco; Le Pont de Claix, France), $1 \mathrm{~g}$ yeast extract (BD Bacto, Le Pont de Claix, France), $5 \mathrm{~g} \mathrm{NaCl}$ (Fisher Scientific, Illkirch, France), $20 \mathrm{~g}$ D-glucose (Fisher Scientific), $1 \mathrm{~g}$ $\mathrm{KH}_{2} \mathrm{PO}_{4}$ (Fisher Scientific) and $15 \mathrm{~g}$ agar granulated (BD Difco) per litre of deionised water. Before sterilising at $121^{\circ} \mathrm{C}$ for $15 \mathrm{~min}$, the $\mathrm{pH}$ of the medium was adjusted to $7.0 \pm 0.2$. The choice of these two strains was due to a strong yellow colour amongst the whole collection of $A$. arilaitensis strains of our laboratory. It was previously shown that all the strains presented the same HPLC pigment profile, including the strain Re117, the genome sequence of which is available (Sutthiwong \& Dufossé, 2014).

After inoculation of $10 \mathrm{~mL}$ of milk ingredient-based liquid medium (same composition as described previously, without agar) in a $50 \mathrm{~mL}$ conical flask with a colony of $A$. arilaitensis, the pre-cultures were incubated for $72 \mathrm{~h}$ at $25^{\circ} \mathrm{C}$ on a rotary shaker at $150 \mathrm{rpm}$. The same growth medium was then inoculated at $1 \%(\mathrm{v} / \mathrm{v})$ with the preculture and incubated under the same conditions.

\subsection{Pigment extraction}

After $7 \mathrm{~d}$ of growth, A. arilaitensis cells were harvested by centrifugation at $6000 \times g\left(25^{\circ} \mathrm{C}\right)$ for $15 \mathrm{~min}$. The cell pellets were washed with deionised water, frozen at $-80^{\circ} \mathrm{C}$ for $48 \mathrm{~h}$, and then lyophilised to dryness (COSMOS-80 manufactured by CRYOTEC, vacuum below 200 mtorr, temperature of the shelf increased to $-20^{\circ} \mathrm{C}$ for overnight primary drying then to $20^{\circ} \mathrm{C}$ for $2 \mathrm{~h}$ for the secondary drying phase). Lyophilised cells $(1 \mathrm{~g})$ were extracted with $8 \mathrm{~mL}$ of 99\% methanol (Carlo Erba, Peypin, France) including a sonication step (Transsonic T420" homogeniser, Elma, Singen, Germany, HF-frequency $35 \mathrm{kHz}, 10 \mathrm{~min}$, on ice), at room temperature for $90 \mathrm{~min}$. The mixture was centrifuged at $6000 \times \mathrm{g}\left(25^{\circ} \mathrm{C}\right)$ for $15 \mathrm{~min}$ to separate the cell debris from the carotenoid-containing supernatant. The cell debris were re-extracted two times with $8 \mathrm{~mL}$ of methanol and twice with $8 \mathrm{~mL}$ of methyl tert-butyl ether (MTBE; Carlo Erba) until complete bleaching of the biomass was obtained. The extracts were combined and subsequently evaporated to dryness under vacuum at $55{ }^{\circ} \mathrm{C}$ using a Büchi Rotavapor (BÜCHI Labortechnik AG, Flawil, Switzerland). The residue was dissolved in $1 \mathrm{~mL}$ methanol/MTBE (1:1, v/v), filtered through Millex-GV 0.2- $\mu \mathrm{m}$ hydrophilic membrane (Millipore, Molsheim, France), and stored at $-20{ }^{\circ} \mathrm{C}$ in an amber vial prior to HPLC analysis.

\subsection{Chromatographic analysis}

Analyses were carried out using a Nexera liquid chromatography (LC) system (Shimadzu, Milan, Italy), consisting of a CBM$20 A^{\mathrm{TM}}$ controller, two LC-30AD ${ }^{\mathrm{TM}}$ dual-plunger parallel-flow pumps, a DGU-20 A5 ${ }^{\mathrm{TM}}$ degasser, a CTO-30A ${ }^{\mathrm{TM}}$ column oven, and an SIL-30A ${ }^{\mathrm{TM}}$ autosampler. A $0.1 \mathrm{~mm}$ internal diameter stainless steel tubing (zero dead volume) was employed for column connection. The LC system was coupled to a liquid chromatography-mass spectrometry ion trap time-of-flight (LCMS-IT-TOF) mass spectrometer (MS) through an atmospheric-pressure chemical ionisation (APCI) source operating in both positive and negative mode (Shimadzu, Kyoto, Japan). Data acquisition was carried out by means of the LCMS solution software (Version 3.50.346; Shimadzu).

Chromatographic separation was achieved on Ascentis Express Fused-core C18 columns, $150 \times 4.6 \mathrm{~mm}$ i.d., $2.7 \mu \mathrm{m}$ d.p., kindly donated by Supelco/Sigma-Aldrich (Bellefonte, PA, USA). The mobile phases consisted of methanol (eluent A) and methyl tertbutyl ether (eluent B). Elution from the column was isocratically carried out from $100 \%$ (A) for 25 min then a gradient was established over the next 45 min with (B) to a final ratio of 70:30 (A:B), followed by a further elution for 15 min with 70:30 (A:B). The column was then returned to the initial conditions and equilibrated over $20 \mathrm{~min}$. The flow rate was $1 \mathrm{~mL} \mathrm{~min}{ }^{-1}$ and the injection volume was $20 \mu \mathrm{L}$. The UV-visible (UV-vis) spectra were 
acquired in the range of $250-600 \mathrm{~nm}$, while the chromatograms were extracted at $440 \mathrm{~nm}$.

LCMS-IT-TOF MS detection parameters were as follows: detector voltage, $1.50 \mathrm{kV}$; interface temperature: $400{ }^{\circ} \mathrm{C}$; curved desolvation line temperature, $250{ }^{\circ} \mathrm{C}$; block heater temperature, $230{ }^{\circ} \mathrm{C}$; nebulising gas flow $\left(\mathrm{N}_{2}\right), 2.5 \mathrm{~L} \mathrm{~min}^{-1}$; ion accumulation time, $30 \mathrm{msec}$; full scan range, $300-800 \mathrm{~m} / \mathrm{z}$; event time, $300 \mathrm{~ms}$; repeat, 3 ; automatic sensitivity control, $70 \%$. For $\mathrm{MS} / \mathrm{MS}$, full scan range, $50-800 \mathrm{~m} / z$; ion accumulation time, $30 \mathrm{~ms}$; collision induced dissociation energy: 50\%. Samples were analysed in triplicate. Carotenoids were identified by their UV-vis spectra, including spectral fine structure, and MS spectra recorded in both positive and negative $\mathrm{APCI}$ ionisation modes, considering the respective elution order.

\section{Results}

The carotenoid extracts obtained from the cheese ripening bacteria A. arilaitensis, Po102 and Stp101, had chromatographic profiles which were similar to those of 14 other A. arilaitensis strains previously investigated (Sutthiwong \& Dufossé, 2014). A typical chromatogram (Fig. 1A) recorded at $440 \mathrm{~nm}$ displayed two major groups of peaks according to the elution time. The first group contains peak 1 , eluting at $9.79 \mathrm{~min}$, then peak 2 eluting approximately 1 min later (Fig. 1A). Peaks 3 and 4 (Fig. 1A) represent the second group, eluted between 16.5 and $17.5 \mathrm{~min}$.

The main carotenoids were identified as all-E-decaprenoxanthin (peak 1, Fig. 1A), all-E-sarcinaxanthin (peak 2, Fig. 1A), 9-Z-decaprenoxanthin (peak 3, Fig. 1A) and 15-Z-decaprenoxanthin (peak 4, Fig. 1A). These molecules have been characterised by both their UV-vis spectra and MS spectra, both in APCI (-) and (+) ionisation modes. Fig. 1 also shows the photodiode array detector (PDA) UV-vis spectra of the identified peaks (Fig. 1B,C,D,E). Interestingly, there is a small hypsochromic shift in $\lambda$ max of 3-7 $\mathrm{nm}$ for the cis isomers compared with the trans isomer for decaprenoxanthin. The appearance of a typical cis absorption band at around $142 \mathrm{~nm}$ below the longest-wavelength absorption maximum was also observed for the 9-Z-decaprenoxanthin (at $318 \mathrm{~nm}$ ) and for 15-Z-decaprenoxanthin (at $320 \mathrm{~nm}$ ), which correspond to peaks 3 and 4 respectively. Moreover, the cis band for the 15-Z-decaprenoxanthin isomer showed a greater intensity being nearer to the centre of the molecule and also showed a double cis-peak appearance, i.e., with two maxima, characteristic of a chromophore bearing a long aliphatic moiety.

The mass spectra of the two principal types of carotenoids biosynthesised by $A$. arilaitensis, i.e., decaprenoxanthin and sarcinaxanthin (shown as 1 and 2, respectively, in Table 1) in APCI (-) and APCI $(+)$ ionisation modes, are reported in Fig. 2. The mass
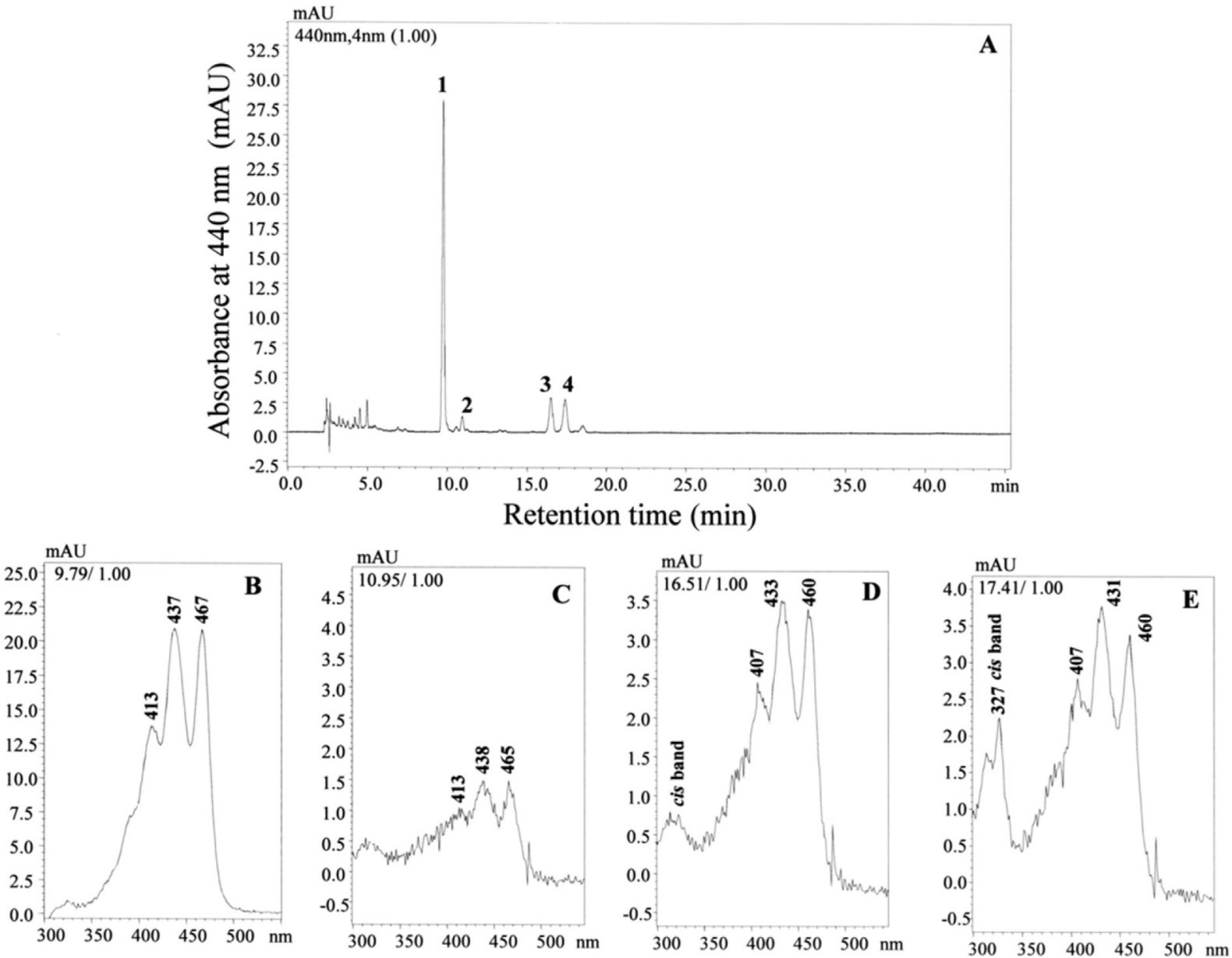

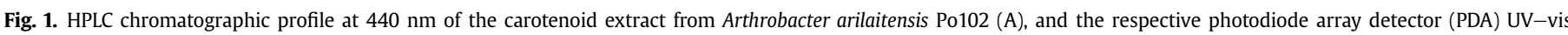

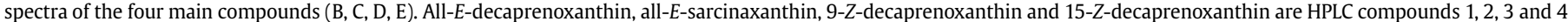

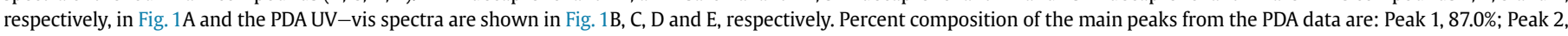
3.5\%; Peak 3, $4.5 \%$ and Peak $4,4.5 \%$. 
Table 1

Identification of the carotenoids in the extracts of Arthrobacter arilaitensis Po102.

\begin{tabular}{|c|c|c|c|c|}
\hline Compound & Identification & UV-vis maxima (nm) & MS data APCI (-) & MS data $\mathrm{APCI}(+)$ \\
\hline SMGp & Sarcinaxanthin monoglucoside pentaacetate & Not detected & 1076 & Not detected \\
\hline DDG & Decaprenoxanthin diglucoside & Not detected & 1028,849 & Not detected \\
\hline DMG & Decaprenoxanthin monoglucoside & Not detected & 866 & Not detected \\
\hline 1 & Decaprenoxanthin & $413,437,467$ & 704 & $705,689,687,669,595$ \\
\hline 2 & Sarcinaxanthin & $415,438,466$ & 704 & $705,687,551$ \\
\hline 3 & 9-Z-decaprenoxanthin & $318,409,434,460$ & 704 & $705,687,669,549,523$ \\
\hline 4 & 15-Z-decaprenoxanthin & $320,407,431,460$ & 704 & $687,669,577$ \\
\hline Dec-C16:0 & Decaprenoxanthin-C16:0 & Not detected & 942 & Not detected \\
\hline
\end{tabular}

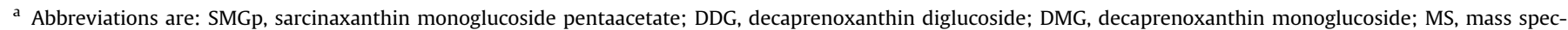
trometry; APCI, atmospheric-pressure chemical ionisation.

spectra clearly show the pseudomolecular radical ion at $m / z 704$ in the negative ionisation mode for decaprenoxanthin (Fig. 2A) and sarcinaxanthin (Fig. 2C). In the positive ionisation mode (Fig. 2B and $\mathrm{D}$ ), the fragment ions produced in the APCI source (i) by the loss of water, and toluene from decaprenoxanthin (Fig. 2B) and (ii) by the loss of water and the fragment at $m / z 136$, from sarcinaxanthin (Fig. 2D), are presented.

The other minor carotenoids, which were present in very low amounts, were only detected in APCI (-) ionisation mode (Table 1). These carotenoids were identified as sarcinaxanthin monoglucoside pentaacetate, decaprenoxanthin diglucoside (DDG), decaprenoxanthin monoglucoside (DMG) and decaprenoxanthin-C16:0 (decaprenoxanthin-palmitate, Dec-C16:0). Fig. 3 shows the superimposed extracted ion chromatograms at $m / z$ 849 (1028-179, for DDG), at $m / z 866$ for DMG, at $m / z 942$ for Dec-C16:0, and at $m / z$ 704 for compounds 1, 2, 3, 4 as reported in Table 1, together with the UV-vis and MS spectra information for the identified compounds. The chemical structures of the two most important carotenoids, from a metabolic point of view, produced by A. arilaitensis: (i) decaprenoxanthin, $\left(2 R, 6 R, 2^{\prime} R, 6^{\prime} R\right)-2,2^{\prime}$-bis-(4hydroxy-3-methylbut-2-enyl)- $\varepsilon, \varepsilon$-carotene; and (ii) sarcinaxanthin, $\left(2 R, 6 R, 2^{\prime} R, 6^{\prime} R\right)-2,2^{\prime}$-bis-(4-hydroxy-3-methylbut-2-enyl)$\gamma, \gamma$-carotene, are shown in Fig. 4 , where the whole picture of the eight carotenoids detected in our research is presented in the framework of a biosynthetic pathway.

\section{Discussion}

In this study, the pigments extracted from the biomass of A. arilaitensis strains originating from cheese were characterised by an HPLC-PDA-APCI-MS methodology. According to the data of the UV-vis spectra, the mass spectra both in APCI $(-)$ and APCI $(+)$ ionisation modes, and the elution order, A. arilaitensis produces eight $C_{50}$ carotenoids. It produces mainly free-form (non-glycosylated) all-E-decaprenoxanthin (carotenoid with $\varepsilon$ ring), and two of the cis isomers, 9-Z-decaprenoxanthin and 15-Z-decaprenoxanthin, in minor amounts. Decaprenoxanthin is also produced as monoand diglucosides, and as an ester with palmitic acid as the acid moiety.

9-Z-decaprenoxanthin and 15-Z-decaprenoxanthin isomers are always in the same proportions within these bacterial pigmented extracts, as analysed in our laboratory over many years, representing over 100 liquid chromatography injections, even before the official description of the new species $A$. arilaitensis was made in the literature by Irlinger et al. (2005), or the in-depth analysis of the chemical structures of the pigments carried out in the present study. Contrary to the many conclusions found in the literature, $Z$ isomers of carotenoids (as observed in this study) are not artefacts. Indeed, these isomers are naturally produced, as shown by Melendez-Martinez, Stinco, Liu, and Wang (2013).
Another interesting result of the present study is the concomitant presence of decaprenoxanthin and derivatives (carotenoids with an $\varepsilon$ ring) and sarcinaxanthin and derivative (carotenoids with a $\gamma$ ring). To the best of our knowledge, this is the first time that the simultaneous presence of these two C50 cycles is described in a non-genetically engineered bacterium. Up to now, sarcinaxanthin biosynthesis was described in $M$. luteus (Netzer et al., 2010) and decaprenoxanthin biosynthesis in $C$. glutamicum (Heider, Peters-Wendisch, \& Wendisch, 2012; Krubasik et al., 2001).

The whole genome sequencing of the strain originating from cheese, Arthrobacter arilaitensis Re117, reveals the presence of genes encoding the following proteins: Idi, CrtE, CrtB, CrtI, CrtEb, CrtYe and CrtYf, whose counterparts in C. glutamicum (from 44 to $54 \%$ identity) catalyse the production of decaprenoxanthin from lycopene (Monnet et al., 2010). Cyclases, such as CrtYeYf, are key enzymes of the $\mathrm{C} 50$ carotenoid biosynthesis pathway and have been investigated using molecular biology (Netzer et al., 2010). The $M$. luteus CrtYgYh polypeptides constitute a $\gamma$-cyclase, which specifically converts flavuxanthin into sarcinaxanthin. Interestingly, it was shown that the $C$. glutamicum CrtYeYf cyclase not only converts flavuxanthin into decaprenoxanthin. Indeed, when expressed in Escherichia coli, CrtYeYf also catalyses the synthesis of sarcinaxanthin and sarprenoxanthin (Netzer et al., 2010). Whether these additional reactions may also occur during the growth of C. glutamicum has not been established. However, we may hypothesise that the simultaneous production of decaprenoxanthin and sarcinaxanthin in A. arilaitensis cells could be due to multiple catabolic activities of its CrtYeYf cyclase.

Carotenoids are synthesised by a large variety of plants, algae and microorganisms. These pigments have several important functional properties, principally antioxidant activity, as well as prevention of certain diseases, such as cancer, cardiovascular and Alzheimer's pathologies (Kirsh et al., 2006; Li, Shen, \& Ji, 2012). Due to their beneficial effects, they have been increasingly used in a wide range of food, pharmaceutic and cosmetic products, as well as in the animal feed industry for livestock, poultry, fish, and crustaceans (Anunciato \& da Rocha Filho, 2012; Pickworth, Loerch, Kopec, Schwartz, \& Fluharty, 2012; Tarique et al., 2013; Wang, Chien, \& Pan, 2006; Yuan, Peng, Yin, \& Wang, 2011). More than 95\% of all natural carotenoids are based on a symmetric C40 phytoene backbone, and only a small number of C30 and even fewer C50 carotenoids have been discovered in nature. C50 carotenoids have multiple conjugated double bonds, and they contain at least one hydroxyl group; both of these features contribute to strong antioxidative properties. Therefore, these carotenoids, which are only produced by microorganisms, may have interesting nutraceutical and pharmaceutical applications.

Besides the conventional use in cheese manufacturing, new information about carotenoids synthesised by the cheese-ripening 
Inten.(x1.000.000)

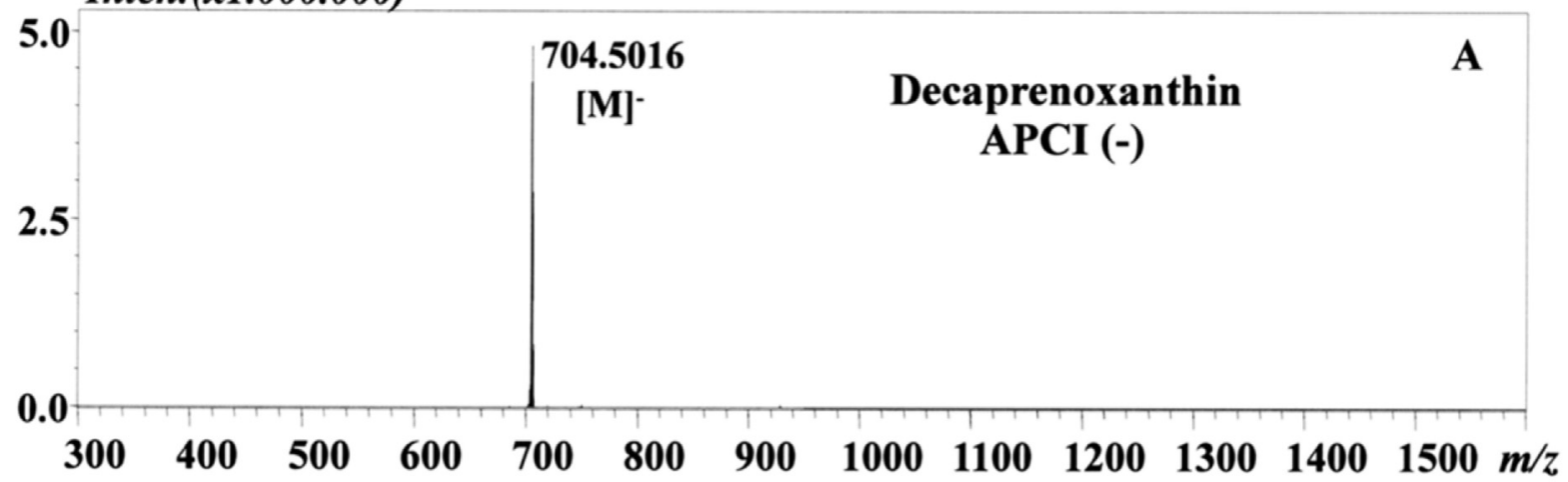

Inten.(x1.000.000)

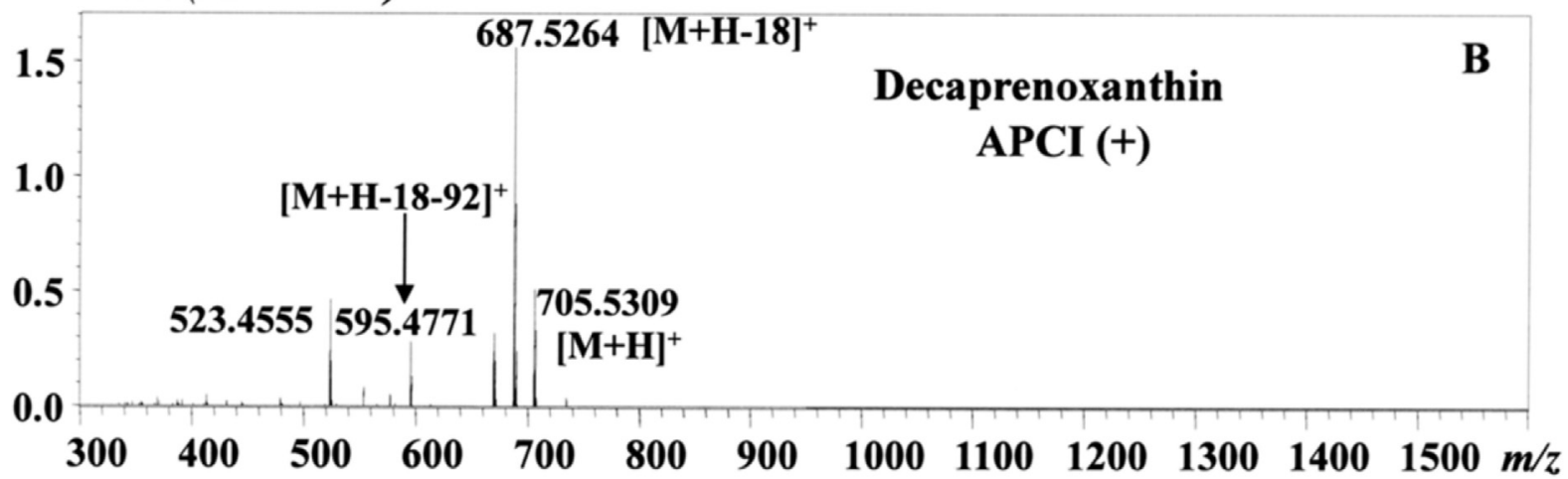

Inten. $(x 10.000)$
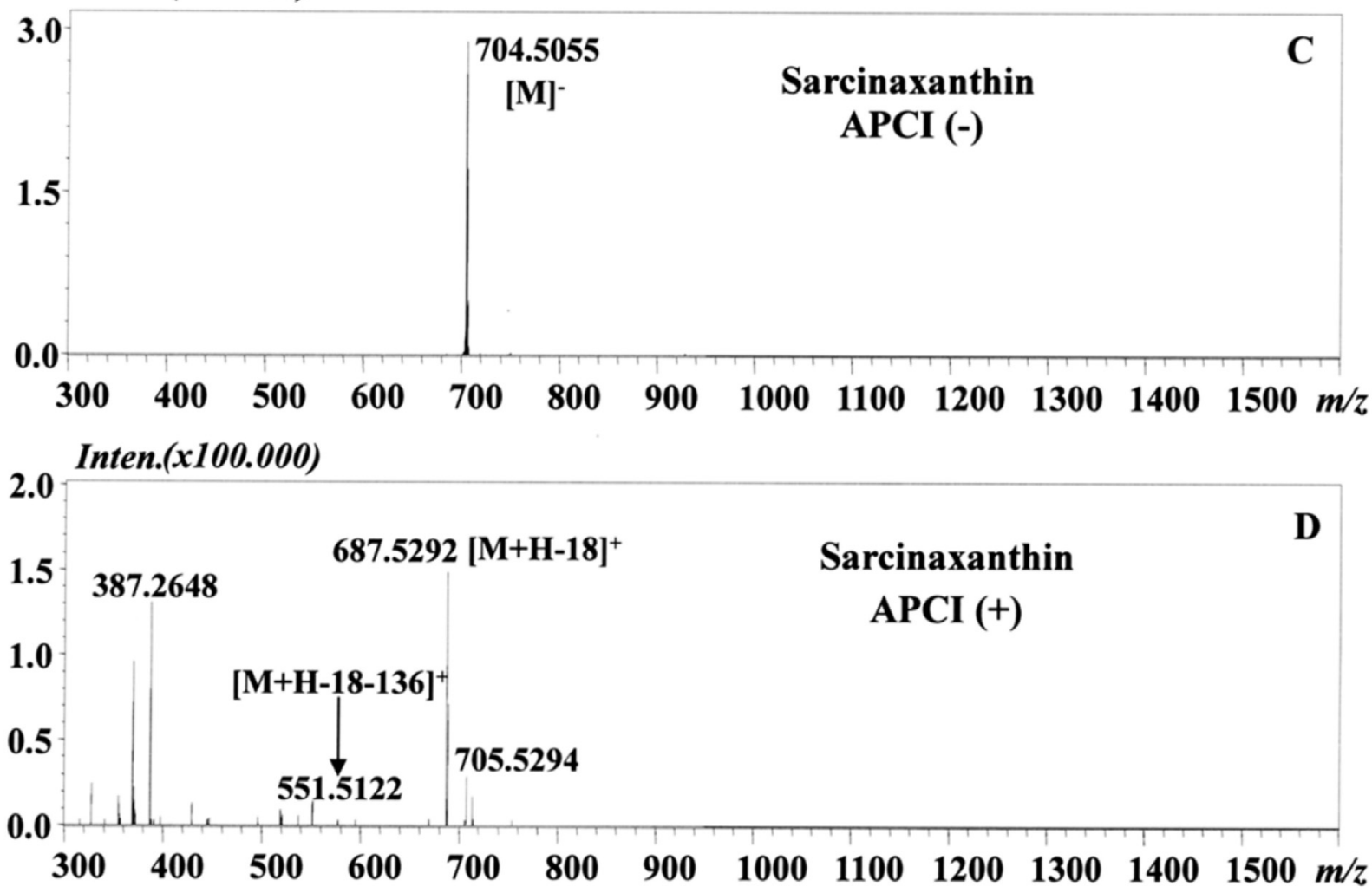

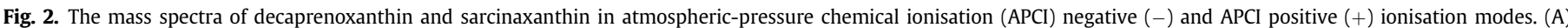

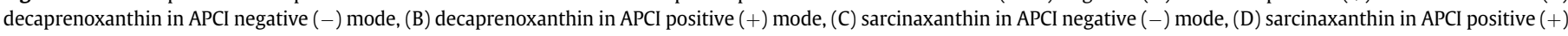
mode. 


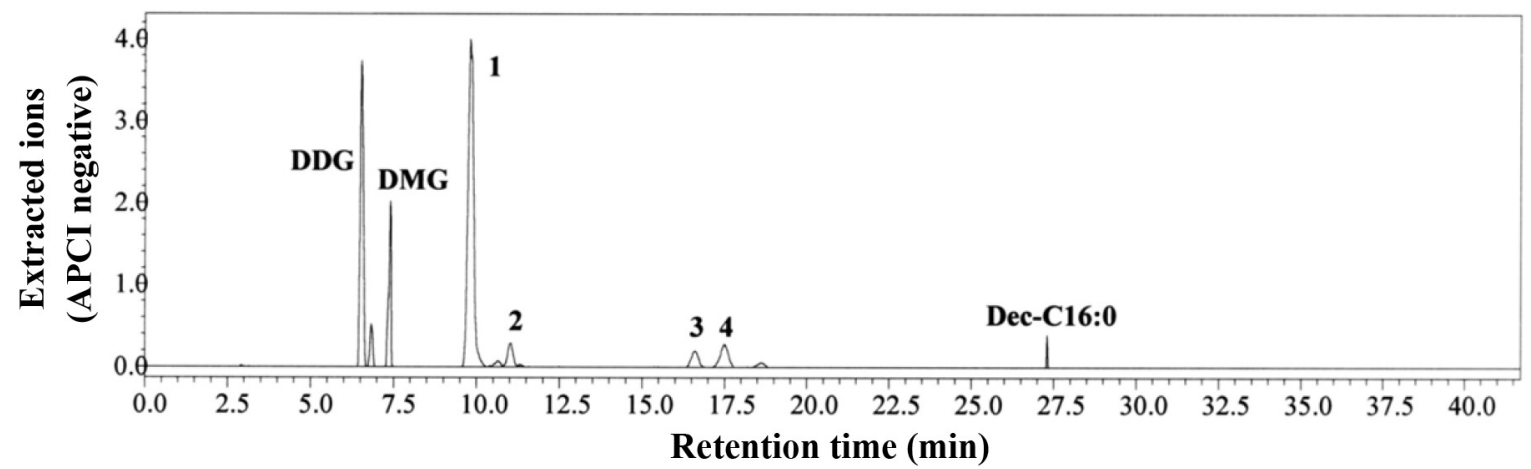

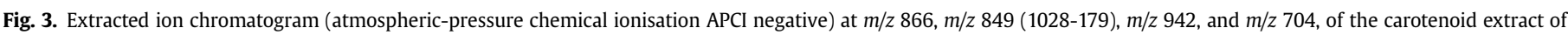
Arthrobacter arilaitensis Po102. DDG: decaprenoxanthin diglucoside; DMG: decaprenoxanthin monoglucoside; Dec-C16:0: decaprenoxanthin-palmitic acid C16:0 ester.

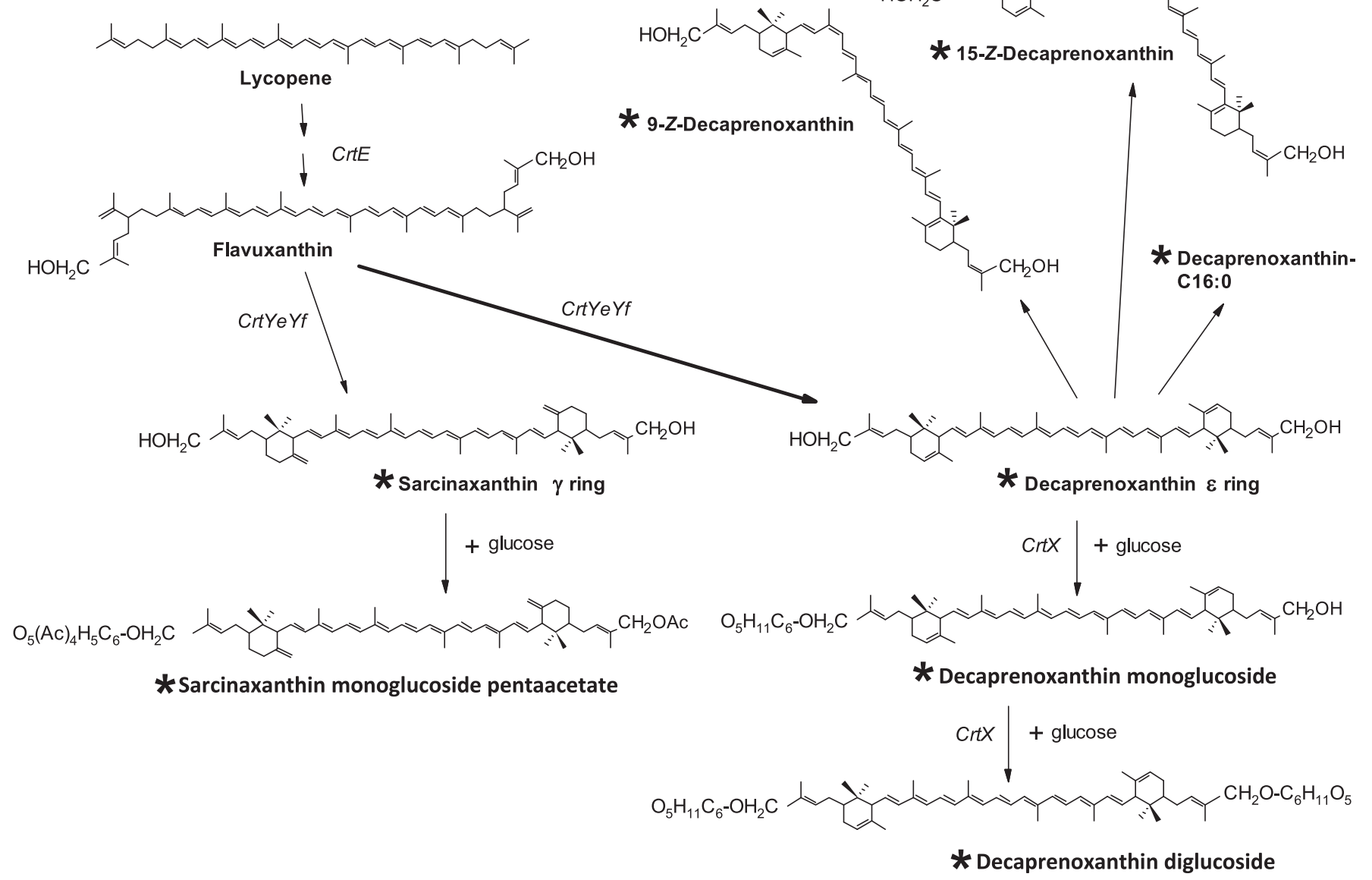

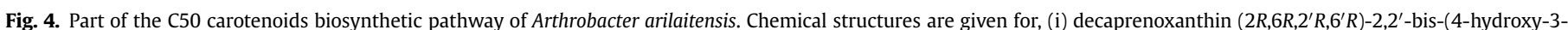

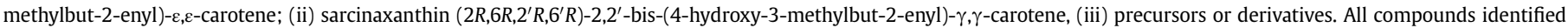
in this study are indicated with a black asterisk against their names. CrtE, CrtYeYf, CrtX are proteins involved in carotenoid biosynthesis.

species, A. arilaitensis, may promote greater acceptability for ingredients extracted from food-grade bacteria, which may lead to an increase in the panel of carotenoids available as food colorants, as well as for other purposes, such as the application of $\mathrm{C}_{50}$ carotenoids in light protecting cosmetics and sunscreens, as these pigments were previously described to be effective UV and visible light filters.

\section{Conclusions}

The pigments extracted from the biomass of cheese-originating A. arilaitensis strains were characterised by an HPLC-PDA-APCI-MS methodology. According to the data of the UV-vis spectra, the mass spectra both in APCI $(-)$ and APCI $(+)$ ionisation modes, and the elution order, $A$. arilaitensis produces eight $C_{50}$ carotenoids. It 
produces mainly free-form (non-glycosylated) all-E-decaprenoxanthin (carotenoid with an $\varepsilon$ ring), and in minor amounts, two of the cis isomers, 9-Z-decaprenoxanthin and 15-Z-decaprenoxanthin. Decaprenoxanthin is also produced as mono- and diglucosides, and as an ester with palmitic acid as the acid moiety. One interesting result of the present study is the concomitant presence of decaprenoxanthin and derivatives (carotenoids with an $\varepsilon$ ring) and sarcinaxanthin and derivative (carotenoids with a $\gamma$ ring). To the best of our knowledge, this is the first time that the simultaneous presence of these two C50 cycles is described in a non-genetically engineered bacterium.

\section{Acknowledgements}

N. Sutthiwong is grateful to the Royal Thai Government for research grant (GVT2010-0042) obtained for preparing her PhD at the University of Reunion Island, France. L. Dufossé, Y. Caro and M. Fouillaud would like to thank the Conseil Régional de La Réunion, Reunion Island, France, for financial support of research activities dedicated to microbial pigments.

\section{References}

Anunciato, T. P., \& da Rocha Filho, P. A. (2012). Carotenoids and polyphenols in nutricosmetics, nutraceuticals, and cosmeceuticals. Journal of Cosmetic Dermatology, 11, 51-54.

Arpin, N., Faisson, J.-L., Norgard, S., Borch, G., \& Liaaen-Jensen, S. (1975). Bacterial carotenoids, XLVI. $C_{50}$-Carotenoids, 14 . $C_{50}$-Carotenoids from Arthrobacter glacialis. Acta Chemica Scandinavica, B29, 921-926.

Arpin, N., Liaaen-Jensen, S., \& Trouilloud, M. (1972). Bacterial carotenoids, XXXVIII $\mathrm{C}_{50}$-Carotenoid 9. Isolation of decaprenoxanthin mono-and di-glucoside from an Arthrobacter sp. Acta Chemica Scandinavica, 26, 2524-2526.

Bockelmann, W., \& Hoppe-Seyler, T. (2001). The surface flora of bacteria smearripened cheese from cows' and goats' milk. International Dairy Journal, 11, 307-314.

Dufossé, L., Mabon, P., \& Binet, A. (2001). Assessment of the coloring strength of Brevibacterium linens strains: spectrocolorimetry versus total carotenoid extraction/quantification. Journal of Dairy Science, 84, 354-360.

Eliskases-Lechner, F., \& Ginzinger, W. (1995). The bacterial flora of surface-ripened chesses with special regard to coryneforms. Lait, 75, 564-571.

Feurer, C., Vallaeys, T., Corrieu, G., \& Irlinger, F. (2004). Does smearing inoculum reflect the bacterial composition of the smear at the end of the ripening of a French soft, red-smear cheeses? Journal of Dairy Science, 87, 3189-3197.

Galaup, P., Flamin, C., Carlet, E., \& Dufossé, L. (2005). HPLC analysis of the pigment produced by the microflora isolated from the 'Protected Designation of Origin' French red-smear soft cheeses Munter, Epoisses, Reblochon and Livarot. Food Research International, 38, 855-860.

Galaup, P., Gautier, A., Piriou, Y., de Villeblanche, A., Valla, A., \& Dufossé, L. (2007). First pigment fingerprints from the rind of French PDO red-smear ripened soft chesses Epoisses, Mont d'Or and Maroilles. Innovative Food Science and Emerging Technologies, 8, 373-378.

Goerges, S., Mounier, J., Rea, M. C., Gelsomino, R., Heise, V., Beduhn, R., et al. (2008). Commercial ripening starter microorganisms inoculated into cheese milk do not successfully establish themselves in the resident microbial ripening consortia of south German red smear cheese. Applied and Environmental Microbiology, 74, 2210-2217.

Heider, S. A. E., Peters-Wendisch, P., Netzer, R., Stafnes, M., \& Brautaset, T. (2014). Production and glucosylation of $\mathrm{C}_{50}$ and $\mathrm{C}_{40}$ carotenoids by metabolically engineered Corynebacterium glutamicum. Applied Genetics and Molecular Biotechnology, 98, 1223-1235.

Heider, S. A. E., Peters-Wendisch, P., \& Wendisch, V. F. (2012). Carotenoid biosynthesis and overproduction in Corynebacteria glutamicum. BMC Microbiology, 12, 198-208.

Irlinger, F., Bimet, F., Delettre, J., Lefèvre, M., \& Grimont, P. A. D. (2005). Arthrobacter bergerei sp. nov. and Arthrobacter arilaitensis sp. nov., novel coryneform species isolated from the srfaces of cheeses. International Journal of Systematic and Evolutionary Microbiology, 55, 457-462.

Irlinger, F., Layec, S., Hélinck, S., \& Dugat-Bony, E. (2015). Cheese rind microbia communities: diversity, composition and origin. FEMS Microbiology Letters, 362, $1-11$.

Irlinger, F., \& Mounier, K. (2009). Microbial interactions in cheese: implications for cheese quality and safety. Current Opinion in Biotechnology, 20, 142-148.

Kirsh, V. A., Mayne, S. T., Peters, U., Chatterjee, N., Leitzman, M. F., Dixon, L. B., et al. (2006). A prospective study of lycopene and tomato product intake and risk of prostate cancer. Cancer Epidermiology, Biomarkers and Prevention, 15, 92-98.

Kohl, W., Achenbach, H., \& Reichenbach, H. (1983). The pigments of Brevibacterium linens: aromatic carotenoids. Phytochemistry, 22, 207-210.

Krubasik, P., Takaichi, S., Maoka, T., Kobayashi, M., Masamoto, K., \& Sandmann, G. (2001). Detailed biosynthetic pathway to decaprenoxanthin diglucoside in Corynebacterium glutamicum and identification of novel intermediates. Archives of Microbiology, 176, 217-223.

Larpin-Laborde, S., Imran, M., Bonaïti, C., Bora, N., Gelsomino, R., Goerges, S., et al (2011). Surface microbial consortia from Livarot, a French smear-ripened cheese. Canadian Journal of Microbiology, 57, 651-660.

Leclercq-Perlat, M.-N., Corrieu, G., \& Spinnler, H.-E. (2004). The color of Brevibacterium linens depends on the yeast used for cheese deacidification. Journal of Dairy Science, 87, 1536-1544.

Leclercq-Perlat, M.-N. \& Spinnler, H.-E. (2010). The type of cheese curds determined the colouring capacity of Brevibacterium linens and Arthrobacter species. Journal of Dairy Research, 77, 1-8.

Li, F. J., Shen, L., \& Ji, H. F. (2012). Dietary intakes of vitamin E, vitamin C, and $\beta$ carotene and risk of Alzheimer's disease: a meta-analysis. Journal of Alzheimer's Disease, 31, 253-258.

Melendez-Martinez, A. J., Stinco, C. M., Liu, C., \& Wang, X.-D. (2013). A simple HPLC method for the comprehensive analysis of cis/trans (Z/E) geometrical isomers of carotenoids for nutritional studies. Food Chemistry, 138, 1341-1350.

Monnet, C., Loux, V., Gilbrat, J.-F., Spinnler, H.-E., Barbe, V., Vacherie, B., et al. (2010) The Arthrobacter arilaitensis Re117 genome sequence reveals its genetic adaptation to the surface cheese. PLoS ONE, 5, e15489.

Montel, M.-C., Buchin, S., Mallet, A., Delbes-Paus, C., Vuitton, D. A., Desmasures, N., et al. (2014). Traditional cheeses: rich and diverse microbiota with associated benefits. International Journal of Food Microbiology, 177, 136-154.

Mounier, J., Gelsomino, R., Goerges, S., Vancanneyt, M., Vandemeulebroecke, K. Hoste, B., et al. (2005). Surface microflora of four smear-ripened cheeses. Applied and Environmental Microbiology, 71, 6489-6500.

Mounier, J. Goerges, S., Gelsomino, R., Vancanneyt, M., Vandemeulebroecke, K, Hoste, B., et al. (2006). Sources of the adventitious microflora of a smearripened cheese. Journal of Applied Microbiology, 101, 668-681.

Mounier, J., Monnet, C. Vallaeys, T., Arditi, R., Sarthou, A.-S., Hélias, A., et al. (2008). Microbial interactions within a cheese microbial community. Applied and Environmental Microbiology, 74, 172-181.

Netzer, R., Stafsnes, M. H., Andreassen, T., Goksøyr, A., Bruheim, P., \& Brautaset, T. (2010). Biosynthetic pathway for $\gamma$-cyclic sarcinaxanthin in Micrococcus luteus: heterologous expression and evidence for diverse and multiple catalytic functions of $\mathrm{C}_{50}$ carotenoid cyclases. Journal of Bacteriology, 192 5688-5699.

Pickworth, C., Loerch, S. C., Kopec, R. E., Schwartz, S. J., \& Fluharty, F. L. (2012) Concentration of pro-vitamin A carotenoids in common beef cattle feedstuffs. Journal of Animal Science, 90, 1553-1561.

Sutthiwong, N., Caro, Y., Milhau, C., Valla, A., Fouillaud, M., \& Dufossé, L. (2014) Arthrobacter arilaitensis strains isolated from ripened cheeses: characterization of their pigmentation using spectrocolorimetry. Food Research International, 65 184-192.

Sutthiwong, N., \& Dufossé, L. (2014). Production of carotenoids by Arthrobacter arilaitensis strains isolated from smear-ripened cheeses. FEMS Microbiology Letters, 360, 174-181.

Tao, L., Yao, H., \& Cheng, O. (2007). Gene from a Dietzia sp, for synthesis of C40 and C50 beta-cyclic carotenoids. Gene, 386, 90-97.

Tarique, T. M., Yang, S., Mohsina, Z., Qiu, J., Zhao, Y., Gang, C., et al. (2013). Role of carotenoids in poultry industry in China: a review. Journal of Natural Science Research, 3, 111-121.

Wang, Y.-J., Chien, Y.-H., \& Pan, C.-H. (2006). Effects of dietary supplementation of carotenoids on survival, growth, pigmentation, and antioxidation capacity of characins, Hyphessobrycon callistus. Aquaculture, 261, 641-648.

Yuan, J. P., Peng, J., Yin, K., \& Wang, J. H. (2011). Potential health-promoting effects of astaxanthin: a high-value carotenoid mostly from microalgae. Molecular Nutrition and Food Research, 55, 150-165. 